\title{
Developing a clinical screening tool for frailty in the acute care setting
}

\author{
Authors: Moe Oo, ${ }^{A}$ Yun Chan, ${ }^{A}$ Helen Chamberlain ${ }^{A}$ and Shu $\mathrm{Ho}^{\mathrm{B}}$
}

\section{Aims}

To develop and validate a simple frailty screening tool that could cover major components of frailty and be applicable as a quick assessment in busy clinical settings.

\section{Methods}

\section{Developing the tool}

A frailty assessment tool was designed, based on evidence from current studies. The tool has five criteria. These include age (75 and over), fall with any injury or fracture (excluding fracture of the neck of the femur), dementia or delirium, care home residence, and reduced or lack of mobility over 24 hours. If any acute elderly inpatient meets at least three of these five criteria, this patient should be diagnosed as having frailty, indicating a need for a comprehensive geriatric assessment. The background rationale for establishing these criteria is as follows.

$>$ Ageing should be seen as a key parameter influencing frailty in older people.

> Fall and fragility fractures are directly proportionate to risk of frailty. However, fracture of the neck of the femur should be excluded, as there is a separate assessment and care pathway for older people with this in hospitals.

$>$ Any kind of cognitive impairment, either acute or chronic, closely links with frailty in old age.

> Prevalence of frailty is very common in institutional-dwelling older people.

$>$ Reduced mobility or physical activities correlate with frailty in the elderly.

\section{Validating the tool}

The inter-observer and intra-observer reproducibility of the tool were evaluated as follows:

> Ten patients, aged 75 years and above, acutely and medically admitted to the hospital were randomly selected. Using the tool, frailty was assessed among these patients. The rate of inter-observer reproducibility was mainly tested. Two different paired observers (two different grades of doctor, a doctor and a physiotherapist) assessed the same patient with the tool.
> Frailty was assessed among ten patients in different clinical settings to evaluate the intra-observer reproducibility. In this assessment, one observer (a doctor) assessed the same patient in two different clinical settings; the emergency department $(\mathrm{A} \& \mathrm{E})$ and the acute medical admission unit (AMU).

> All observers involved did not contribute to developing the tool. They were all independent observers.

> The validation of the tool was conducted on 1-14 March 2012 in Good Hope Hospital, Heart of England NHS Foundation Trust.

\section{Results}

In terms of the inter-observer reproducibility of the tool, eight out of a total of ten patients $(80 \%)$ were given the same frailty scores, whereas the other two patients (20\%) were given different scores by only 'one mark', relating to the criterion 'reduced or lack of mobility in last 24 hours'. This parameter may be scored by direct observation of patients, or self-reporting by patients or carers, thus causing either observer or participant bias. However, these potential biases were only associated with one out of five parameters on the whole scale, and therefore the overall validity of the scale was not altered significantly. Regarding the intra-observer reproducibility of the tool, ten out of a total of ten patients (100\%) received the same frailty score, proving that different clinical settings did not change the validity of the screening tool.

\section{Conclusions}

Our frailty screening tool showed satisfactory outcomes in terms of testing its inter-observer and intra-observer reproducibility. Thus it is generally justified that this tool could be applicable in assessing frailty. Further multicentred validation should be conducted to prove its generalisability.

\section{Conflict of interest statement}

This is our own work. No direct patient contact was involved. There was no other conflict of interest in this work. 